\title{
Vortex-Induced Vibrations of a Low-tension Cable-Sheave System Modeled using Nonlinear Finite Elements
}

\author{
Cassidy Westin, Rishad A. Irani \\ Department of Mechanical and Aerospace Engineering \\ Carleton University \\ Ottawa, Canada \\ cassidy.westin@carleton.ca; rishad.irani@ carleton.ca
}

\begin{abstract}
This work presents a finite element model of a cablesheave system constructed to model cable vibrations due to vortex shedding in low-tension cases. The study assesses the potential for vortex shedding to lead to detachment of the cable from the sheave. The absolute nodal coordinate formulation is utilized to define the cable structural dynamics. Vortex shedding forces are incorporated by coupling the cable's equations of motion to a Van der Pol equation, also known as a wakeoscillator and the cable-sheave interaction is described using a contact penalty method. The study examines the contribution of vortex shedding occurring at the cable's natural frequency to the cable motion. For the cases studied the model demonstrates that vortex shedding alone results in very small vibration amplitudes and thus is unlikely to result in cable detachment.
\end{abstract}

Keywords-finite element method; absolute nodal coordinate formulation; cable-sheave interaction; vortex-induced vibration

\section{INTRODUCTION}

Cable-sheave systems are commonly used in marine applications for towing and lifting. The cable is subjected to highly dynamic loading due to the ship motion, hydrodynamic forces and wind loading which can result in the cable falling off the sheave. Cables exposed to external fluid flow experience vortex induced vibrations (VIV) resulting from the periodic shedding of vortices in the cables wake. The vortex shedding produces oscillating lifting and drag forces on the cable, which can excite a resonance response in the cable if the shedding frequency matches the fundamental frequency of the cable. Excessive cable vibrations can result in wear of both the cable and the sheave and possible detachment of the cable from a sheave. It is thus useful to quantify the vibration amplitudes due to wind loading using a dynamic model and assess the potential for vortex shedding to lead to cable detachment.

Gabbai and Benaraoya [1] give an overview of the various modeling approaches used to predict the response of cylinders to the vortex shedding forces. One modelling approach known as a wake-oscillator model utilizes a second order differential equation, such as the Van der Pol equation, to represent the flow in the wake. The oscillator is coupled with the structural dynamics of the cable using forcing terms in both equations. This modelling approach does not require numerical modeling of the flow field and can be easily incorporated into existing dynamic cable models. Existing studies of cable vibrations using wake oscillator models typically utilize continuous equations to represent the cable structural dynamics. The contribution of the current research is the coupling of a wake-oscillator model and a finite element cable model, which has not been extensively studied.

This paper presents the construction of a finite element model using the absolute nodal coordinate formulation (ANCF). The model, used for a planar cable-pulley system in the researchers previous work [2], has been extended to include three dimension contact with the sheave grooves. Additionally, oscillating lift and drag forces using an existing wake-oscillator model [3] have been incorporated. Cases of low cable tension are considered as cable detachment is more likely to occur when the cable is slack. High sustained wind velocities are neglected in the study and only the contribution of vortex shedding at the cable's natural frequency is considered. Section 2 of the paper describes the cable model and the formulation of the external forces. Case studies examining the potential for VIV to lead to cable detachment are presented in Section 3. The paper ends with concluding remarks in Section 4.

\section{Finite Element And External LoAd Model}

The finite element model is comprised of $N$ two node cable elements. Each node has 6 degrees of freedom consisting of a position vector and a vector tangent to the cable centerline. All degrees of freedom are defined in the absolute coordinate frame. An additional parameter $p \in[0, l]$ is used to interpolate 
between nodes. The absolute coordinates $\mathbf{r}$ of a point on the cable is defined using a vector of generalized coordinate $\mathbf{q}$ and a cubic shape function $\mathbf{S}(p)$ which interpolates between the nodes of each element:

$$
\mathbf{r}(p)=\mathbf{S}(p) \mathbf{q}=\left[\begin{array}{lll}
x & y & z
\end{array}\right]^{T} .
$$

For a fully parameterized ANCF beam element of unstretched length $l$, the generalized coordinates consist of the Cartesian coordinates $\mathbf{r}$ and the parametric slopes $\partial \mathbf{r} / \partial p$ at each node:

$$
\mathbf{q}=\left[\begin{array}{llll}
\mathbf{r}_{1}{ }^{T} & (\partial \mathbf{r} / \partial p)_{1}{ }^{T} & \mathbf{r}_{2}{ }^{T} & (\partial \mathbf{r} / \partial p)_{2}{ }^{T}
\end{array}\right]^{T}
$$

where subscripts 1 and 2 represent the respective nodes of the element or parameter values of $p=0$ and $p=l$, respectively. The shape function representing a cubic spline is

$$
\mathbf{S}(p)=\left[\begin{array}{c}
\left(1-3(p / l)^{2}+2(p / l)^{3}\right) \mathbf{I} \\
\left((p / l)-2(p / l)^{2}+(p / l)^{3}\right) \mathbf{I} \\
\left(3(p / l)^{2}-2(p / l)^{3}\right) \mathbf{I} \\
\left((p / l)^{2}-(p / l)^{3}\right) \mathbf{I}
\end{array}\right]^{T}
$$

where $\mathbf{I}$ is a $3 \times 3$ identity matrix.

The standard form of the Newton-Euler equations are given for a single element as

$$
\mathbf{M} \ddot{\mathbf{q}}+\mathbf{K q}+\mathbf{C} \dot{\mathbf{q}}-\mathbf{Q}=0,
$$

where $\mathbf{M}$ is the mass matrix, $\mathbf{K}$ is the elastic stiffness matrix, $\mathbf{C}$ is the damping matrix, $\mathbf{Q}$ is an external force vector, and $\mathbf{q}$ is the vector of generalized coordinates defined in Equation 2. The equations of motion for the full system are formulated from Equation 4 using the embedding technique described in [4]. The Newton-Euler equations are solved using a numerical ODE integrator to determine the cable motion over time. The matrices $\mathbf{M}, \mathbf{K}$ and $\mathbf{C}$ and force vector $\mathbf{Q}$ will be defined in the following sections.

\section{A. Mass Matrix}

Using a variational mass lumping approach the mass matrix is derived directly from the element kinetic energy [5] and is given by

$$
\mathbf{M}=\frac{\partial^{2} E_{T}}{\partial \dot{\mathbf{q}} \partial \dot{\mathbf{q}}}=\int_{0}^{l} \rho A \mathbf{S}(\mathbf{p})^{T} \mathbf{S}(\mathbf{p}) d p
$$

where $E_{T}$ is the kinetic energy of the element, $\rho$ is the cable density and $A$ is the cable cross-sectional area.

\section{B. Stiffness}

Similarly, the stiffness matrix is derived from the strain energy $E_{S}$ of the element given by [6]:

$$
E_{S}=\frac{1}{2} \int_{0}^{l}\left[E A \epsilon_{l}^{2}(p)+E I \kappa^{2}(p)\right] d p
$$

where $E$ is the Young's modulus of the cable material, $A$ is the cross-sectional area, $I$ is the second moment of area, $\epsilon_{l}$ is the longitudinal strain, and $\kappa$ is the curvature of the element. The internal stiffness force is then given by the derivative of the strain energy with respect to the coordinate vector $\mathbf{q}$. The force vector can by separated into longitudinal and transverse components, $\mathbf{Q}_{l}$ and $\mathbf{Q}_{t}$. In order to simplify the computation of these forces it is assumed that the longitudinal strain $\epsilon_{l}$ is small and also constant throughout the element. The forces can then be derived as shown by Berzeri et al. [6]:

$$
\mathbf{Q}_{\mathbf{l}}=\left[E A \epsilon_{l} \int_{0}^{l} \mathbf{S}^{\prime T} \mathbf{S}^{\prime} d p\right] \mathbf{q}
$$

where $\mathbf{S}^{\prime}$ is the derivative of $\mathbf{S}$ with respect to the parameter $p$. The longitudinal strain is determined from the arc length $s$ of the element [2]:

$$
\epsilon_{l}(\mathbf{q})=\frac{s(\mathbf{q})-l}{l}
$$

where $s$ is the arc length and $l$ is the unstretched element length. The arc length is defined by integrating the norm of $\partial \mathbf{r} / \partial p$ with respect to $p$ over the length of the element [7]. The arc length $s$ is approximated using a numerical quadrature

$$
s(\mathbf{q})=\sum_{i=1}^{N_{I}} w_{i} \sqrt{\mathbf{r}^{\prime}\left(p_{i}\right)^{T} \mathbf{r}^{\prime}\left(p_{i}\right)} \frac{l}{N_{I}}
$$

where $i$ denotes the integration point, $N_{I}$ is the total number of integration points, $w_{i}$ is the quadrature weight. The trapezoidal rule is used with quadrature weights defined

$$
w_{i}= \begin{cases}0.5, & i=1, N_{I} \\ 1, & i=2,3, \ldots, N_{I}-1\end{cases}
$$

The transverse stiffness force $\mathbf{Q}_{t}$ is defined [6]

$$
\mathbf{Q}_{t}=\left[\int_{0}^{l} E I \mathbf{S}^{\prime \prime T} \mathbf{S}^{\prime \prime} d p\right] \mathbf{q}
$$

where $I$ is the second moment of area. The general stiffness matrix $\mathbf{K}$ from Equation 4 is then given by

$$
\mathbf{K}=\int_{0}^{l} E I \mathbf{S}^{\prime \prime} \mathbf{S}^{\prime \prime} d p+\epsilon_{l}(\mathbf{q}) E A \int_{0}^{l} \mathbf{S}^{\prime T} \mathbf{S}^{\prime} d p .
$$

\section{Damping}

The damping matrix $\mathbf{C}$ from Equation 4 serves both to include energy dissipation and attenuate high frequency vibrational modes. The cable model uses the Rayleigh-damping approach [8], wherein the damping matrix is defined as a linear combination of the mass and stiffness matrices:

$$
\mathbf{C}=\alpha \mathbf{M}+\beta \mathbf{K}
$$

where $\alpha$ and $\beta$ are scalar damping coefficients. The massproportional damping term represents external viscous damping while the stiffness-proportional damping term represents internal frictional damping. Since the external fluid damping 
can be incorporated into the aerodynamic drag force, the massproportional term will be neglected by setting $\alpha$ equal to zero.

Given a specified damping ratio $\xi$, the corresponding value of the remaining coefficient $\beta$ is given by [9]

$$
\beta=2 \xi \frac{1}{\omega_{n}}
$$

where $\omega_{n}$ is the natural frequency of the cable.

\section{External Forces}

The vector of external forces $\mathbf{Q}$ includes the gravitational body force, the normal contact forces along the sheave surfaces and the aerodynamic loading. The formulation of the forces is provided in the following sections.

1) Contact Force: In order to model the cable-sheave interaction, a contact penalty is used. The cable is allowed to "penetrate" the sheave surface and the normal force os defined as a function of the of the relative penetration $\delta$. The normal force per unit length $\mathbf{f}_{N}$ acting at a single point on the element is defined using the Hunt-Crossley model [10], which represents the surface as a non-linear spring-damper:

$$
\mathbf{f}_{N}=k_{N} \delta^{n}(1+D \dot{\delta}) \mathbf{u}_{n}
$$

where $\mathbf{u}_{n}$ is the unit vector normal to the sheave surface at the point of contact, $k_{N}$ is the contact stiffness, $\delta$ is the relative "penetration" of the node into the surface, $D$ is a damping coefficient and $n$ is a positive constant with a value between 1 and 1.5 from the Hertz contact theory [11]. In the present analysis, a contact stiffness of $1.5 \times 10^{7} \mathrm{~N} / \mathrm{m}$ and a value of $n$ of 1.5 is used based on the values used by Bulin et al [12] and the researchers' previous work [2].

The radius of curvature of the groove is assumed to be equal to the radius of the cable. Two surfaces are defined that are parallel to the straight walls of the sheave groove and intersect at the center of curvature of the groove. In the cross-section shown in Figure 1, the two surfaces are straight solid lines and the sheave groove is a dotted line. If the cable centerline lies below either of the solid lines, a penalty force is produced proportional to the penetration of the cable node into the surface. If the cable node lies above both lines, no normal force is applied. The contact penalty is illustrated in Figure 1. The normal force per unit length is evaluated at $N_{I}$ discrete points points per element. The distributed force in the element coordinates is then calculated from the discrete force distribution that will be discussed in Section II-D4.

2) Drag Force: The drag force per unit length acting at a given point on the cable is given by [13]

$$
\mathbf{f}_{D}=-\frac{1}{2} C_{D} \rho_{w} d\left|\mathbf{V}_{n}\right| \mathbf{V}_{n}
$$

where $C_{D}$ is the drag coefficient, $d$ is the cable diameter, $\mathbf{V}_{n}$ is the normal component of the relative velocity $V$, and $\mu$ is the dynamic viscosity of the fluid. The relative velocity and its normal component are calculated as follows:

$$
\mathbf{V}=\dot{\mathbf{r}}-\mathbf{U}
$$

$$
\begin{gathered}
\mathbf{V}_{t}=\left(\mathbf{V} \cdot \mathbf{u}_{t}\right) \mathbf{u}_{t} \\
\mathbf{V}_{n}=\mathbf{V}-\mathbf{V}_{t}
\end{gathered}
$$

where $\mathbf{U}$ is the velocity of the free stream, $\dot{\mathbf{r}}$ is the velocity of the point on cable centerline. The unit vector tangent to the cable centerline $\mathbf{u}_{t}$ is equivalent to $\frac{\mathbf{r}^{\prime}}{\left|\mathbf{r}^{\prime}\right|}$. The drag coefficient $C_{D}$ is given by

$$
C_{D}=1.1+4 R e^{0.8}, \quad 30<\operatorname{Re}<10^{5}
$$

where $R e$ is the Reynolds number.

3) Wake Oscillator Model: A two-degree of freedom wakeoscillator developed by Bai and Qin [3] is used to describe the vortex shedding forces on the cable. The variable $w(t)$ is introduced, with the function $d^{2} \dot{w}(t)$, where $d$ is the cable diameter, corresponding to the vortex strength of the wake. The evolution of $w$ over time satisfies a van der Pol equation of the form

$$
\ddot{w}+a_{1} \omega_{s t}\left[1-\frac{a_{2}(\dot{w})^{2}}{\omega_{s t}^{2}}\right] \dot{w}+\omega_{s t} w=\frac{a_{3} \omega_{s t}}{d} \dot{Y}
$$

where $a_{1}, a_{2}$ and $a_{3}$ are empirical parameters, $\omega_{s t}$ is the Strouhal frequency in rad/s, and $Y$ is the displacement of the cable in the cross-flow direction. Typical values for $a_{1}, a_{2}$ and $a_{3}$, proposed by Facchinetti [14] and Bai and Qin [3] are 0.3, 0.2 and 12 , respectively.

The forces acting on the structure due to the vortex shedding are then evaluated:

$$
\begin{gathered}
f_{S W}=-\frac{C_{D 0} \rho d^{4}}{32 \pi^{3} S t^{3} U} \dot{w} \ddot{w} \\
f_{C F}=\frac{C_{L 0} \rho U d^{2}}{8 \pi S t} \dot{w}
\end{gathered}
$$

where $f_{S W}$ is the force per unit length acting in the streamwise direction, $f_{C F}$ is the force per unit length acting in the cross-flow direction, $C_{D 0}$ and $C_{L 0}$ are the drag and lift coefficients associated with the vortex shedding, with assumed values of 0.2 and 0.3 , respectively. In the absolute coordinate frame, the cross-flow direction corresponds to the y-axis, while the stream-wise direction corresponds to the z-axis.

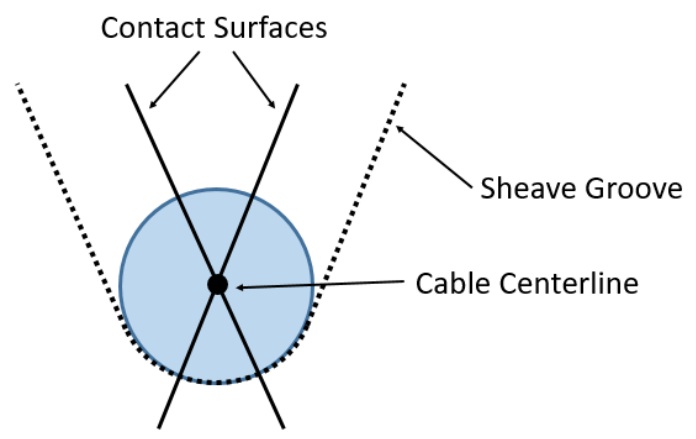

Fig. 1: Sheave Groove Cross-Section 
For each node on the cable exposed to the wind, a degree of freedom representing the wake variable $w$ is added to the system equations of motion. The wake coefficients are interpolated linearly to provide the discrete force distributions along each element.

4) Total Distributed Force: The force distributions determined for the normal contact, stationary drag and oscillating drag and lift are added together to give the total force distribution $\mathbf{f}$. The force acting on the element in the element coordinates $\mathbf{Q}$ is then found by integrating over the length of the cable element:

$$
\mathbf{Q}=\int_{0}^{l} \mathbf{S}(p)^{T} \mathbf{f} d p .
$$

Again, a numerical quadrature is used to approximate the integral:

$$
\mathbf{Q}=\sum_{i=1}^{N_{I}} w_{i} \mathbf{S}(p)^{T} \mathbf{f}_{i} \frac{l}{N_{I}}
$$

where $i$ denotes the integration point, $N_{I}$ is the total number of integration points and $w_{i}$ is the quadrature weight. Ten integration points per element were used for all simulations described in this report.

The gravitational body forces are defined similarly with $\mathbf{f}=$ $\left[\begin{array}{lll}0 & -\rho A g & 0\end{array}\right]^{T}$ where $\rho$ is the cable density and $g$ is the acceleration due to gravity. Since the gravitational force is constant, the integration can be performed symbolically using Equation 21 prior to the simulation.

\section{CASE Studies}

\section{A. Model Parameters}

The system consists of a cable suspended by two identical sheaves. Each cable end is attached to a point mass as shown in Fig. 2. The wind vector is aligned with the z-axis. Static drag and oscillating lift and drag are applied only to the portion of the cable suspended between the two sheaves. A number of case studies were performed. The system properties used for the simulations are listed in Table I. The cable properties used are for a wire rope with $6 \times 37$ construction, commonly used for marine lifting and hoisting applications [15]. The sheave diameters are calculated using the minimum recommended sheave-to-rope diameter ratio of 18 [15].

Values for the cable bending stiffness $E I$ and damping ratio $\xi$ are not readily available, thus conservative values were used. A minimum bending stiffness value can be calculated by neglecting the friction between individual wires and assuming the total bending stiffness is the sum of the bending stiffnesses of the individual wires [16]. Using the average diameter $d_{a v g}$ and assuming the lay angle $\theta$ is small, the bending stiffness becomes

$$
E I=E \sum \frac{\pi d_{w}{ }^{4}}{64} \cos \theta=E N_{w} \frac{\pi d_{a v g}{ }^{4}}{64}
$$

where $N_{w}$ is the number of wires. Based on the parameters listed in Table I, the bending stiffness is estimated to be 0.73
TABLE I: Model parameters.

\begin{tabular}{|l|c|}
\hline Cable diameter, $d$ & $20 \mathrm{~mm}$ \\
Cable mass per unit length, $\mu$ & $1.38 \mathrm{~kg} / \mathrm{m}$ \\
Damping ratio, $\xi$ & 0.10 \\
Cable elastic modulus, $E$ & $75.8 \mathrm{GPa}$ \\
Cable bending stiffness, $E I$ & $0.73 \mathrm{Nm}^{2}$ \\
Cable metallic area, $A$ & $1.64 \times 10^{-4} \mathrm{~m}^{2}$ \\
Sheave radius at root, $R$ & $0.18 \mathrm{~m}$ \\
Sheave groove angle & 30 degrees \\
Sheave groove depth & $30 \mathrm{~mm}$ \\
\hline
\end{tabular}

TABLE II: Case study parameters.

\begin{tabular}{|c|c|c|c|c|}
\hline Case & 1 & 2 & 3 & 4 \\
\hline Span length (m) & 2 & 5 & 5 & 5 \\
Cable Tension (N) & 200 & 200 & 500 & 1000 \\
Natural frequency (rad/s) & 19.9 & 7.57 & 12.0 & 16.9 \\
Wind velocity (m/s) & 0.302 & 0.121 & 0.190 & 0.269 \\
\hline
\end{tabular}

$\mathrm{Nm}^{2}$. Damping ratios for wire ropes undergoing bending vary with cable tension and have been shown to be as high as $37 \%$ for slack cables [17]. A conservative damping ratio of $10 \%$ was used for the simulations.

The natural frequency of the catenary is estimated by treating the cable as a thin Euler-Bernoulli beam pinned at both ends and is given by [18]

$$
\omega_{n}=\left(\frac{\pi}{L}\right)^{2} \sqrt{\frac{E I}{m}\left[1+\frac{T}{E I}\left(\frac{L}{\pi}\right)^{2}\right]}
$$

where $L$ is the beam length, $m$ is the mass per unit length, $T$ is the axial tension, and $E I$ is the bending stiffness. The vortex shedding frequency can be calculated using the Strouhal relation [3]:

$$
f_{S t}=\frac{S t U}{d}=\frac{\omega_{S t}}{2 \pi}
$$

where $S t$ is the Strouhal number, assumed to have a value of 0.2 [14], $U$ is the free stream velocity and $d$ is the cable diameter.

Simulations were performed for three cases; the model parameters specific to each case are given in Table II. In each case, the wind velocity was chosen such that the shedding frequency matches the estimated natural frequency of the cable.

\section{B. Mesh Convergence}

Fig. 2 shows how the cable was divided into five segments: the two free cable ends with point masses, the two segments in contact with the sheaves and the catenary suspended between the sheaves. The wind load is applied only to the catenary, segment 3. Each segment was further divided into elements. A smaller element length is desirable for the elements in contact with the sheave as the curvature is greater. A mesh convergence was performed by allowing the system to reach 


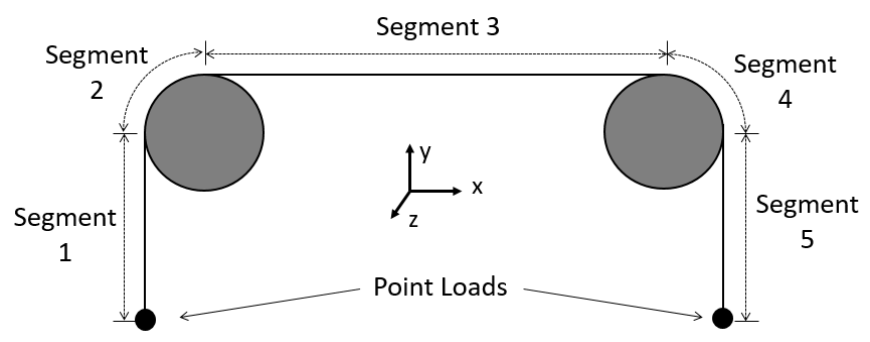

Fig. 2: Diagram of cable-sheave system. Air flow is along the $\mathrm{z}$-axis.

equilibrium with no wind load applied and iteratively reducing the length of the elements in the contact arc and comparing the contact force at the top of the sheave. The number of elements was found to have a minimal effect on the contact force.

For segment 3, the convergence is determined by simulating 20 seconds of motion with the wind load applied. The maximum amplitudes of the motion at the mid-point of the span were compared. Convergence was considered to occur when the change in the output was less than $1 \%$. Fig. 3 plots the mesh convergence for the vertical amplitudes of the 2 and $5 \mathrm{~m}$ spans. For the final simulations, 5 elements were used for each of the contact segments while 12 and 16 elements were used for the 2 and $5 \mathrm{~m}$ cable spans. The free cable ends were assumed not to undergo significant transverse motion, thus only 2 elements were used for each.

\section{Results}

For each case in Table II, the motion was simulated until a steady oscillation was obtained. Fig. 4 displays the vertical motion at midpoint of the span the for Case 4. The results for each test case are shown in Table III. The maximum amplitude at the midpoint of the span was $4.5 \times 10^{-6} \mathrm{~m}$ for Case 4 . At the sheave, the maximum amplitude was $3.3 \times 10^{-6} \mathrm{~m}$. The amplitude of the motion is much smaller than the $30 \mathrm{~mm}$ sheave groove depth.

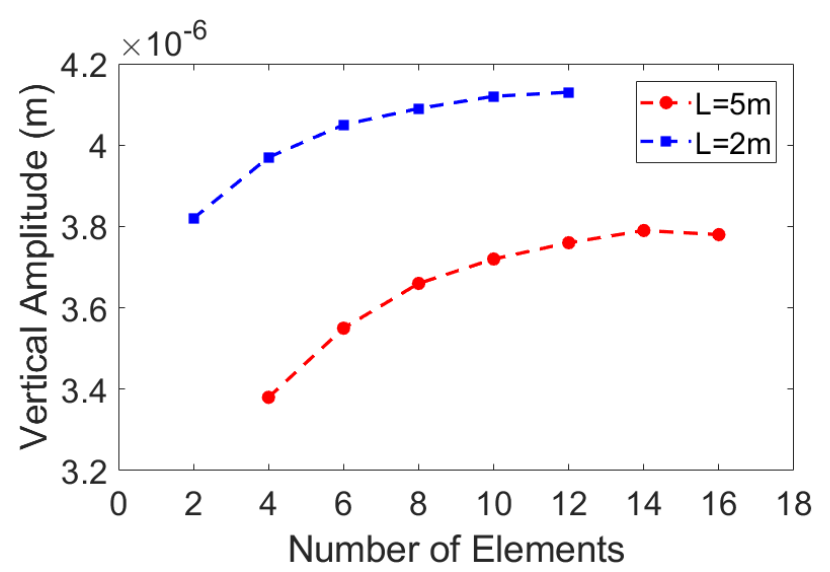

Fig. 3: Convergence curves for segment 3
TABLE III: Simulated vibration amplitudes.

\begin{tabular}{|c|c|c|c|c|}
\hline Case & 1 & 2 & 3 & 4 \\
\hline Vertical, Mid-span $\left(10^{-6} \mathrm{~m}\right)$ & 4.1 & 3.8 & 4.4 & 4.5 \\
Streamwise, Mid-span $\left(10^{-6} \mathrm{~m}\right)$ & 0.30 & 0.35 & 0.30 & 0.30 \\
Vertical, Sheave $\left(10^{-6} \mathrm{~m}\right)$ & 0.48 & 1.38 & 3.3 & 2.3 \\
\hline
\end{tabular}

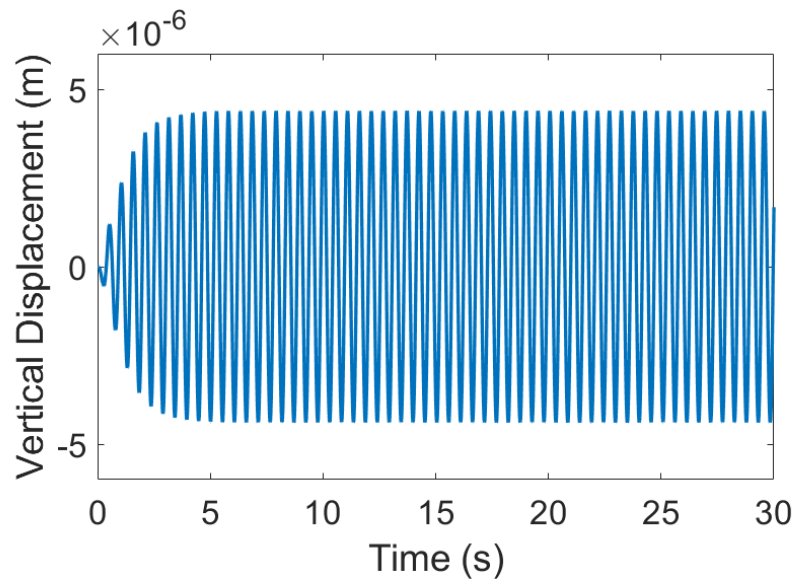

Fig. 4: Mid-span vertical displacement for Case 4

\section{CONCLUSION}

In this paper a dynamic finite element model of a lowtension cable sheave system was used to simulate the vortexinduced vibration of the cable due to wind loading and assess the potential for wind loading to lead to detachment of the cable from the sheave. The model, constructed using the absolute nodal coordinate formulation, utilized a wake-oscillator model to describe the vortex shedding forces. Also, three-dimensional contact with the sheave grooves was introduced. Several case studies were performed for systems of varying span length and cable tension with the wind velocity selected to excite the fundamental mode of the cable. The vibration amplitudes were determined to be small - the maximum amplitude observed at the sheave was only $3.3 \times 10^{-6} \mathrm{~m}$ - thus wind loading alone is unlikely to lead to cable detachment. In future work, the wake-oscillator parameters can be tuned based on wind tunnel experiments. Scenarios where more severe vibrations are likely, such as cables with ice accretion, can also be examined in this manner. Further analysis can be performed to assess the effects of ship motion and higher sustained wind speeds on the response.

\section{ACKNOWLEDGMENT}

The authors would like to thank the Natural Sciences and Engineering Research Council of Canada (NSERC), Kraken Robotic Systems Inc., Rolls-Royce Canada Limited and Carleton University for their support of this research.

\section{REFERENCES}

[1] R. Gabbai and H. Benaroya, "An overview of modeling and experiments of vortex-induced vibration of circular cylinders," Journal of Sound and Vibration, vol. 282, no. 3, pp. 575-616, 2005. 
[2] C. Westin and R. A. Irani, "Cable-pulley interaction with dynamic wrap angle using the absolute nodal coordinate formulation," in Proceedings of the 4th International Conference of Control, Dynamic Systems, and Robotics, 2017.

[3] X. Bai and W. Qin, "Using vortex strength wake oscillator in modelling of vortex induced vibrations in two degrees of freedom," European Journal of Mechanics-B/Fluids, vol. 48, pp. 165-173, 2014.

[4] A. A. Shabana, "Forms of the dynamic equations," Computational Dynamics, Third Edition, pp. 177-210.

[5] A. A. Shabana, "Flexible multibody dynamics: review of past and recent developments," Multibody system dynamics, vol. 1, no. 2, pp. 189-222, 1997.

[6] M. Berzeri and A. A. Shabana, "Development of simple models for the elastic forces in the absolute nodal co-ordinate formulation," Journal of Sound and Vibration, vol. 235, no. 4, pp. 539-565, 2000.

[7] M. Ciftci, Flexible multibody analysis using Absolute Nodal Coordinate Formulation. $\mathrm{PhD}$ thesis, MS Thesis, Middle East Technical Univ., Ankara, Turkey, 2014.

[8] L. Kübler, P. Eberhard, and J. Geisler, "Flexible multibody systems with large deformations and nonlinear structural damping using absolute nodal coordinates," Nonlinear Dynamics, vol. 34, no. 1-2, pp. 31-52, 2003.

[9] I. Chowdhury and S. P. Dasgupta, "Computation of rayleigh damping coefficients for large systems," The Electronic Journal of Geotechnical Engineering, vol. 8, no. 0, pp. 1-11, 2003.

[10] U. Lugrís, J. Escalona, D. Dopico, and J. Cuadrado, "Efficient and accurate simulation of the cable-pulley interaction in weight-lifting machines," in 1st Joint International Conference on Multibody System Dynamics, 2010.

[11] P. Flores, J. Ambrósio, J. P. Claro, and H. Lankarani, "Influence of the contactimpact force model on the dynamic response of multi-body systems," Proceedings of the Institution of Mechanical Engineers, Part K: Journal of Multi-body Dynamics, vol. 220, no. 1, pp. 21-34, 2006.

[12] R. Bulín, M. Hajžman, and P. Polach, "Nonlinear dynamics of a cable-pulley system using the absolute nodal coordinate formulation," Mechanics Research Communications, vol. 82, pp. 21-28, 2017.

[13] S. Takehara, Y. Terumichi, and K. Sogabe, "Motion of a submerged tether subject to large deformations and displacements," Journal of System Design and Dynamics, vol. 5, no. 2, pp. 296-305, 2011.

[14] M. L. Facchinetti, E. De Langre, and F. Biolley, "Coupling of structure and wake oscillators in vortex-induced vibrations," Journal of Fluids and structures, vol. 19, no. 2, pp. 123-140, 2004.

[15] "U.S. Navy wire-rope handbook volume 1," Tech. Rep. ADA955305, Naval Sea Systems Command, 1976.

[16] K. Spak, G. Agnes, and D. Inman, "Cable parameters for homogenous cable-beam models for space structures," in Dynamics of Civil Structures, vol. 4, pp. 7-18, Springer, 2014.

[17] Z. Zhu and S. Meguid, "Nonlinear fe-based investigation of flexural damping of slacking wire cables," International Journal of Solids and Structures, vol. 44, no. 16, pp. 5122-5132, 2007.

[18] N. Al-Raheimy, "Free vibration of simply supported beam subjected to axial force," J Babylon Univ Eng Sci, vol. 20, no. 1, pp. 301-315, 2012. 